\title{
Impact of mutual coupling between SKALA4.1 antennas to the spectral smoothness response
}

\author{
Pietro Bolli $\odot,{ }^{\text {a,* } * \text { Mirko Bercigli } \odot, ~}{ }^{\mathrm{b}}$ Paola Di Ninni, ${ }^{\mathrm{a}}$ Lorenzo Mezzadrelli $\odot,{ }^{\mathrm{c}}$ \\ and Giuseppe Virone ${ }^{\text {d }}$ \\ ${ }^{a}$ Osservatorio Astrofisico di Arcetri, Istituto Nazionale di Astrofisica, Florence, Italy \\ bIngegneria dei Sistemi, Pisa, Italy \\ ${ }^{\mathrm{c}}$ Sirio Antenne, Mantova, Italy \\ ${ }^{\mathrm{d}}$ Istituto di Elettronica e di Ingegneria dell'Informazione e delle Telecomunicazioni, \\ Consiglio Nazionale delle Ricerche, Turin, Italy
}

\begin{abstract}
One of the advantages of arrays with aperiodic distributed elements is their ability to mitigate the detrimental mutual coupling effects on the radiation pattern. However, we show that the mutual coupling inside a random array can still generate undesired structures in the frequency response although the single antenna features a spectral smooth response. For small subsets (a couple of SKALA4.1 antennas and a 16-element array) of a low-frequency instrument station of the Square Kilometre Array, the combination of large mutual coupling and antenna geometry creates systematic distortions in the element frequency responses. This phenomenon compromises the station spectral smoothness response versus frequency. However, we demonstrate that it is possible to partially mitigate these frequency structures by reconfiguring the antenna distribution based on exclusion zones. () The Authors. Published by SPIE under a Creative Commons Attribution 4.0 International License. Distribution or reproduction of this work in whole or in part requires full attribution of the original publication, including its DOI. [DOI: 10 .1117/1.JATIS.8.1.011023]
\end{abstract}

Keywords: log periodic antennas; planar arrays; mutual coupling; radio astronomy.

Paper 21106SS received Sep. 24, 2021; accepted for publication Feb. 8, 2022; published online Feb. 25, 2022.

\section{Introduction}

The spectral smoothness of the station directivity response is a key feature of the low-frequency instrument of the Square Kilometre Array (SKA1-Low). ${ }^{1}$ This requirement arises from the study of the Cosmic Dawn and the Epoch-of-Reionization, which are some of the most significant science cases of SKA1-Low. The redshifted signal from the 21-cm Hydrogen line can be separated from the (much brighter) foreground emission leveraging on their different spectral properties, and this motivates the requirement for a spectrally smooth antenna response. ${ }^{2}$

SKA1-Low is an interferometer of 512 stations operating between 50 and $350 \mathrm{MHz}$ that is currently under construction in Western Australia. Each station is composed by 256 dualpolarized log-periodic dipole antennas (called SKALA4. $1^{3}$ ) installed over a metallic ground plane. The antennas are randomly distributed in a circular area with a maximum distance between two antennas equal to $38 \mathrm{~m}$.

The spectral smoothness demand is quite challenging for a receiving system covering 7:1 relative bandwidth. From an antenna point of view, the intrinsically wide-band log-periodic antenna has been identified, since the early stage of the SKA project, as the most appropriate for providing stable characteristics in the frequency band of interest. ${ }^{4}$

However, there are several factors that cause a reduction in the spectral flatness properties of the SKALA4.1 antenna. First, the metallic ground plane creates a reflected field component, which is added to the direct field radiated by the antenna. The frequency-dependent combination of the two contributions produces a smooth large-scale ripple in the directivity response (see Fig. 7 of Ref. 3). This phenomenon can be accurately modeled with EM solvers. On the other hand, rapid variations of the antenna input impedance, especially at a low frequency,

*Address all correspondnece to Pietro Bolli, pietro.bolli@inaf.it 
compromise the smoothness properties of the transducer gain in a less predictable way. This problem is addressed in Ref. 5, in which a modification of the antenna geometry and the matching network of the low-noise amplifier (LNA) were proposed. Moreover, other narrow-band glitches (0.2 dB peak-to-peak) spaced $\sim 25$ to $30 \mathrm{MHz}$ appear in the SKALA4.1 directivity frequency response from $160 \mathrm{MHz}$ on due to the presence of second-harmonic resonances in the bottom dipoles. ${ }^{6}$

To the best knowledge of the authors, however, in the framework of SKA1-Low, the impact of the mutual coupling between antennas ${ }^{7}$ to the station spectral smoothness properties has been only partially analyzed. In particular, Trott et al. ${ }^{8}$ assessed the calibration performance of SKA1Low in realistic conditions (i.e., including mutual coupling) but only at four discrete frequencies, whereas in Ref. 9, preliminary results were presented for the spectral smoothness for two embedded element patterns of a SKA1-Low prototype system between 70 and $73 \mathrm{MHz}$. It is important to notice that, especially at low frequency, mutual coupling is particularly critical for SKA1-Low due to the large dimension of the SKALA4.1 antenna (maximum horizontal length $1.6 \mathrm{~m}$ ) and to the dense distribution of the antennas. ${ }^{10-12}$ The reason for the lack of a highly sampled spectral analysis is likely due to the heavy computational demand to simulate one SKA1-Low station. For instance, the full-wave analysis of 256 SKALA4.1 antennas by means of the multilevel fast multipole method along with the method of moment technique requires on the order of days for each frequency point on a multicore workstation. ${ }^{13}$ Alternative techniques to accelerate the solution of Maxwell equations in the context of SKA1-Low were proposed in Ref. 14. The numerical analysis proposed in this paper (based on the EM solvers FEKO from Altair and Galileo EMT from Ingegneria dei Sistemi) was first conducted for two SKALA4.1 antennas and then extended to a substation (tile) composed of 16 SKALA4.1 antennas.

This paper is organized as follows. Section 2 shows the mutual coupling effects for two SKALA4.1 antennas placed at different reciprocal distances and alignments, and it gives some physical insights on the phenomenon. Then in Sec. 3, the spectral smoothness results for a tile of 16 antennas are discussed for two different layouts. Finally, Sec. 4 summarizes the conclusions of this study.

\section{Mutual Coupling Between Two SKALA4.1 Antennas}

The SKALA4.1 antenna, selected as the reference antenna for the SKA1-Low project, consists of a 50-ohm active dual-polarized log-periodic antenna with 20 dipoles for each polarization. ${ }^{3}$ Despite this antenna being designed to reach a smooth variation of the directivity response across frequencies, the mutual coupling partially deteriorates this characteristic. In this paper, the zenith directivity is used as a figure of merit to quantify this phenomenon. A relevant example is given by studying two adjacent SKALA4.1 antennas. The zenith directivity of the $X$-polarization port of the antenna located in the origin, SKALA4.1 \#1, is computed while varying the position of the second antenna, SKALA4.1 \#2 (see Fig. 1). The latter is first located at $x=1.5 \mathrm{~m}, y=0 \mathrm{~m}$ (almost touching the other antenna) and then either translated along the $X$ direction (from 1.5 to $2.7 \mathrm{~m}$, SKALA4.1 \#2a in Fig. 1) or moved along a circular trajectory, i.e., constant distance from the origin $(1.5 \mathrm{~m})$, from 0 deg to $45 \mathrm{deg}$ maintaining the same orientation (SKALA4.1 \#2b in Fig. 1). In this analysis, the second antenna is mostly located in the $E$ plane of the excited antenna because, for small antennas interdistances, this plane features larger mutual coupling effects than the $H$ plane. The not-excited ports of the antennas are closed on a 50-ohm load corresponding to the input impedance of the LNA. The presence of these loads takes into account the power coupled by the passive ports and transmitted to the receiver. Finally, the numerical results are computed by laying the antennas on an infinite perfect electric conducting ground plane.

The results of the two series of simulations are summarized in Figs. 2(a) and 2(b) for the circular trajectory and linear shift, respectively. For each geometrical configuration, the spectral behavior of the zenith directivity is very different from the flat directivity $(\sim 8 \mathrm{dBi})$ computed for the isolated SKALA4.1 antenna (see the dashed red curve). In particular, two frequency regions, centered at 55 and $77 \mathrm{MHz}$ and each a few MHz wide, show very distorted behaviors. Apart from

J. Astron. Telesc. Instrum. Syst. 011023-2 Jan-Mar 2022 • Vol. 8(1) 


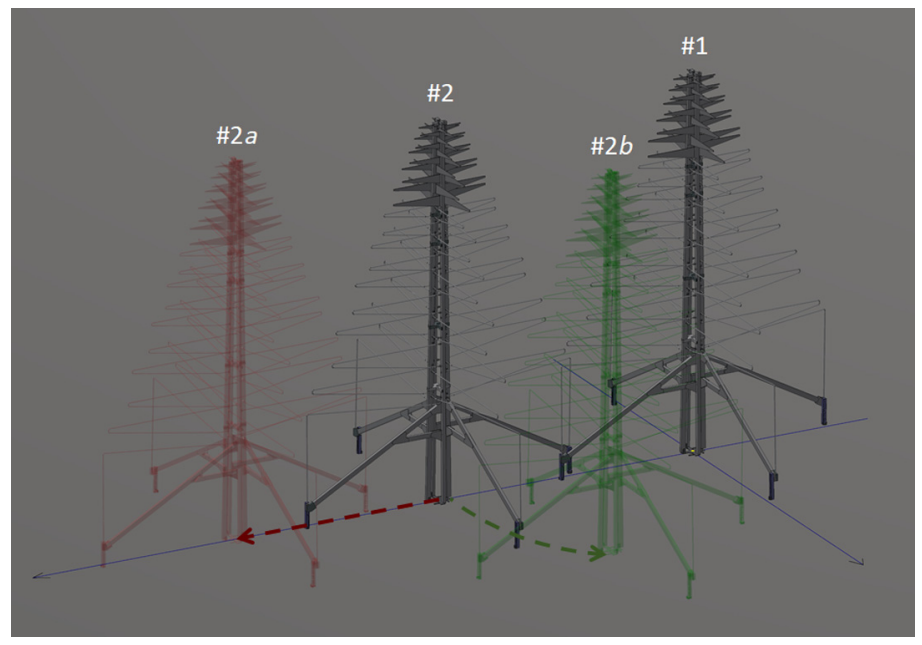

Fig. 1 Geometry of the model with two SKALA4.1 antennas. The antenna in the origin (\#1) is fixed, whereas the position of the second antenna (\#2) varies either along the $X$ axis (up to $2.7 \mathrm{~m}$, \#2a) or along a circular trajectory centered in the origin with a radius of $1.5 \mathrm{~m}$ (up to $45 \mathrm{deg}, \# 2 b$ ). The colored arrows and the transparent SKALA4.1 antennas describe the different extremes of the two movements of the second antenna.

some exceptions [see, for instance, Fig. 2(a), in which the response for the 5-deg alignment is more distorted than at $0 \mathrm{deg}$ ], the bandwidth and the sharpness of directivity in the two frequency regions decrease by increasing the antennas separation or by moving the second antenna away from the $E$ plane. Figure 2 also shows that the resonances never disappear; however, when either the two antennas are aligned in the $E$ plane at a distance larger than $2 \mathrm{~m}$ or they are at $1.5 \mathrm{~m}$ distance but moved away from $E$ plane by more than $25 \mathrm{deg}$, their shape is significantly reduced (all of these configurations are represented in Fig. 2 by the blue color curves).

A physical explanation for this phenomenon is here discussed for the configuration with the two antennas aligned in the $E$ plane and $1.5 \mathrm{~m}$ apart. First, the scattering parameters were analyzed in two narrow frequency regions centered around the two glitches, 53 to 57 and 75 to 79 MHz. Figure 3 shows, in these two frequency ranges, the directivity at zenith for the $X$-polarization port of the antenna located in the origin and its reflection coefficient $\left(S_{11}\right)$. In Fig. 3, the coupling coefficient $\left(S_{21}\right)$ between the two $X$-polarization ports of SKALA4.1 \#1 and \#2 is also reported. The coupling with the orthogonal $Y$-polarization ports is not considered here as it is always lower than that between the parallel ports. The sharp resonances at 55 and $77 \mathrm{MHz}$ are visible in both the zenith directivity and $S$-parameters (see Fig. 3). This phenomenon is due to an abrupt variation of the currents in the antenna (see below) that occurs at the resonance frequencies. In particular, in the 55-MHz region [Fig. 3(a)], the antenna exhibits a strong mismatch of the excited port $\left(S_{11}\right.$ reaching almost $0 \mathrm{~dB}$ ) and consequently a minimum of the $S_{21}$ curve. Around $77 \mathrm{MHz}$ [see Fig. 3(b)], the antenna is always reasonably well matched, whereas the $S_{21}$ curve shows a sharp transition around $77.5 \mathrm{MHz}$ from -5 to $-15 \mathrm{~dB}$. However, the deep null in the zenith directivity at $77 \mathrm{MHz}$ does not correspond to any particular feature in the mutual coupling coefficient. As expected, the scattering parameters do not provide a direct explanation of the glitches in the pattern.

A better insight is instead given by the analysis of the radiation patterns of the SKALA4.1 \#1 coupled to SKALA4.1 \#2 at two critical frequencies, i.e., where the drop of the zenith directivity is maximum ( 54.5 and $77 \mathrm{MHz}$ ), and, for reference, at two very close frequencies slightly outside the resonance (57 and $78 \mathrm{MHz}$ ). The four patterns are reported in Fig. 4(a). They are highly distorted with pointing direction out-of-zenith at the critical frequencies, while at only few $\mathrm{MHz}$ apart they resemble the isolated SKALA4.1 pattern. For comparison, Fig. 4(b) shows the patterns at the same frequencies as obtained for a single SKALA4.1 antenna, which has very smooth behavior across frequencies.

For the same frequencies, the full 3D patterns of the SKALA4.1 \#1 antenna in the presence of the SKALA4.1 \#2 antenna in Fig. 5 emphasize the fully distorted pattern at 54.5 and $77 \mathrm{MHz}$ 

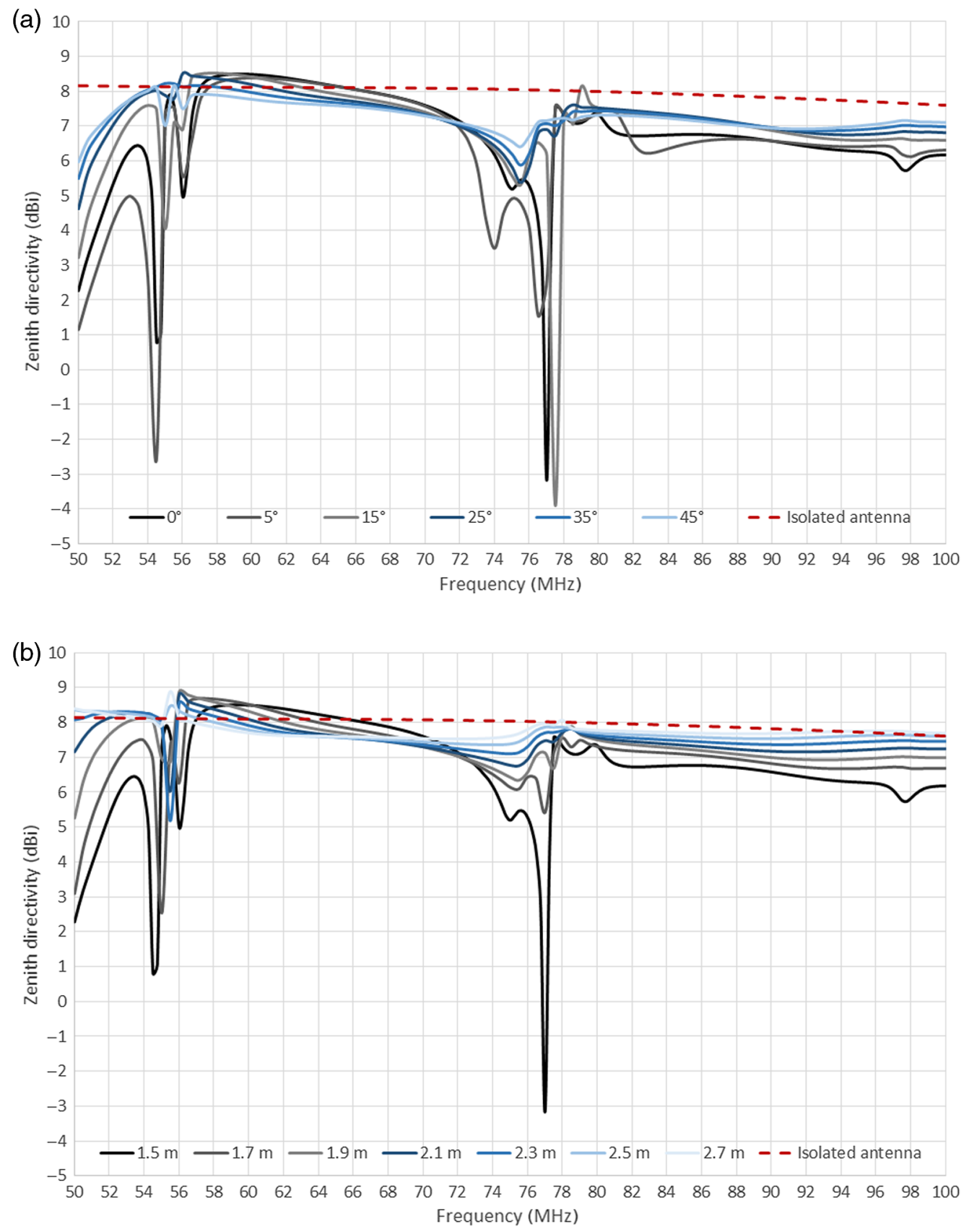

Fig. 2 Directivity at zenith in the frequency band 50 to $100 \mathrm{MHz}$ for the $X$-polarized SKALA4.1 \#1 antenna centered in the origin. The continuous curves correspond to a different position of the second antenna (SKALA4.1 \#2) as described in Fig. 1, whereas the dashed red curve is the isolated antenna response. (a) The case of circular trajectory and (b) the linear shift.

compared with the results at 57 and $78 \mathrm{MHz}$. It is apparent that, at the resonance frequencies (glitches at zenith), the energy is radiated to other directions such as toward the horizon [Fig. 5(a)] and at 45 deg from zenith [Fig. 5(c)].

Finally, Fig. 6 shows the normalized distribution of the electrical currents in the two antennas (SKALA4.1 \#1 excited on the right side and SKALA4.1 \#2 passive on the left side). Again, the current distribution is shown at the critical frequencies and at the other two reference frequencies for comparison. Looking at Fig. 6(a), we notice that at $54.5 \mathrm{MHz}$ the currents are induced only in half of the bottom bow-tie dipole, whereas they are well balanced in both sides of the same dipole at $57 \mathrm{MHz}$ [Fig. 6(b)]. A similar situation occurs also in the other frequency region; Fig. 6(c) shows that at $77 \mathrm{MHz}$ the currents are quite unbalanced in the bottom dipole of the excited 

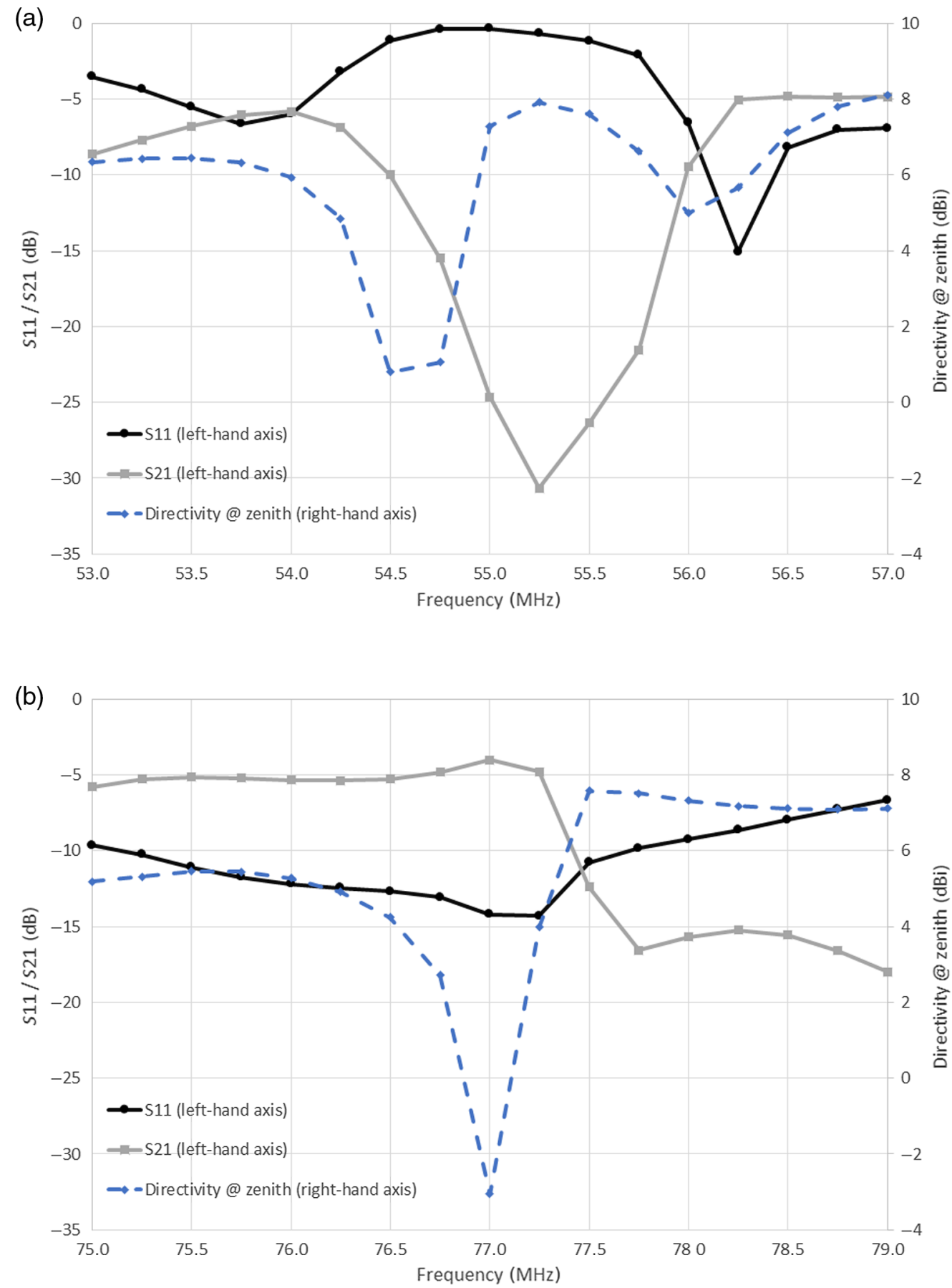

Fig. 3 Directivity at zenith, reflection coefficient, and mutual coupling coefficient for the $X$-polarized SKALA4.1 \#1 antenna with a second antenna SKALA4.1 \#2 located at $X=1.5 \mathrm{~m}$ and $Y=0 \mathrm{~m}$. (a) The frequency bands 53 to $57 \mathrm{MHz}$ and (b) 75 to $79 \mathrm{MHz}$.

antenna and very much coupled to the nearby parasitic antenna. On the other hand, at $78 \mathrm{MHz}$, the currents are reasonably well-distributed in the two sides of the bottom dipoles of the active antenna producing a more regular pattern. In other words, at both the observed resonances (glitches), the adjacent antenna operates as a load that produces a current unbalance on the bottom bow-tie element of the excited SKALA4.1 antenna. This is consistent with the results shown in Fig. 2, which show that glitches are less significant when the bottom bow-tie arms are more separated from each other. This idea has driven the layout optimization strategy presented in the following. 

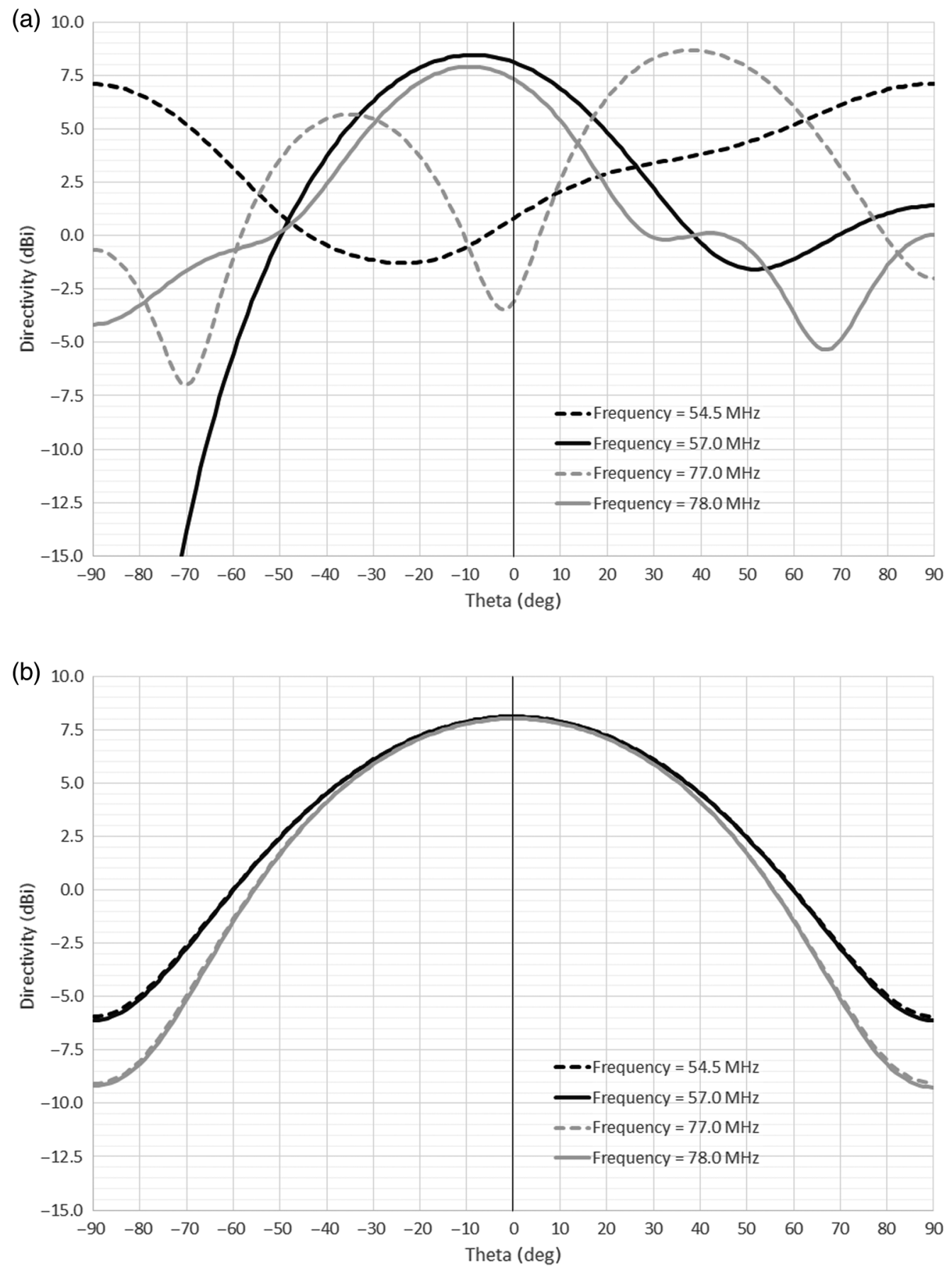

Fig. 4 Antenna patterns in the $E$ plane for the $X$-polarized SKALA4.1 $\# 1$ antenna for two different scenarios: (a) in the presence of the SKALA4.1 \#2 located at $X=1.5 \mathrm{~m}$ and $Y=0 \mathrm{~m}$ and (b) isolated. The patterns are shown at four different frequencies: 54.5 and $77 \mathrm{MHz}$ corresponding to the minimum directivity at zenith and at 57 and $78 \mathrm{MHz}$ used as reference.

\section{Spectral Smoothness Analysis for 16 SKALA4.1 Antennas}

In this section, the electromagnetic analysis is conducted for a tile of 16 antennas. The antenna distribution is based on the SKA1-Low prototype named Aperture Array Verification System ver. 2 (AAVS2).${ }^{15}$ In particular, we selected a tile positioned in the outer part of the station, the layout of which is shown in Fig. 7(a). This tile features a minimum distance between two antennas equal to $1.53 \mathrm{~m}$ (antennas \#13 and \#14) and a maximum distance of $9.87 \mathrm{~m}$ (\#7 and \#12). 
(a)

(b)

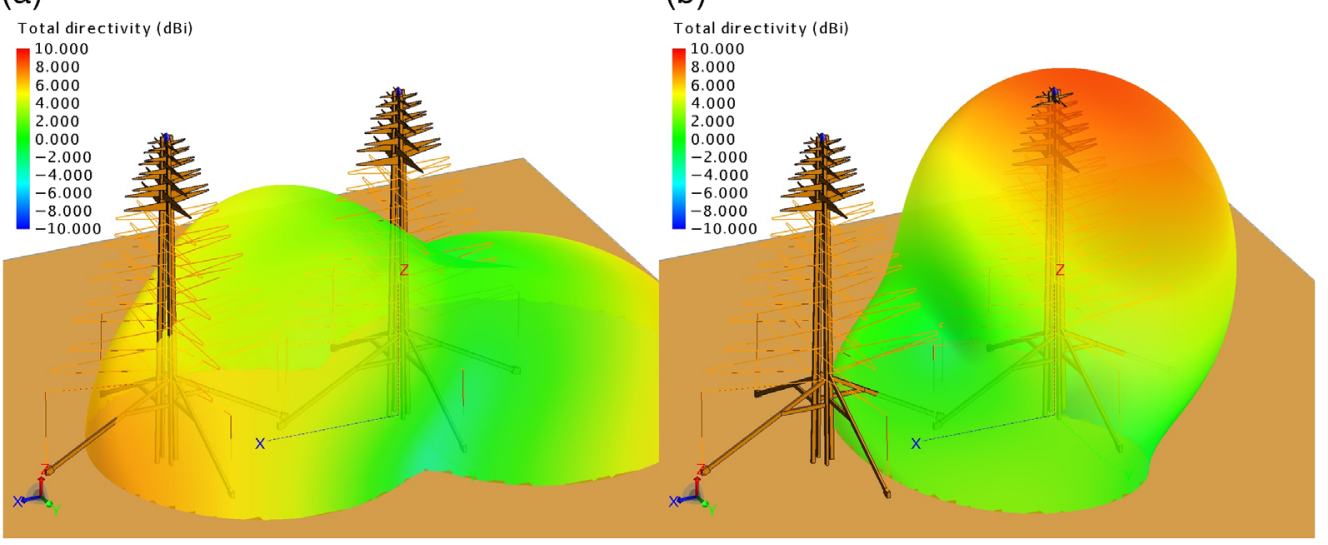

(c)

(d)

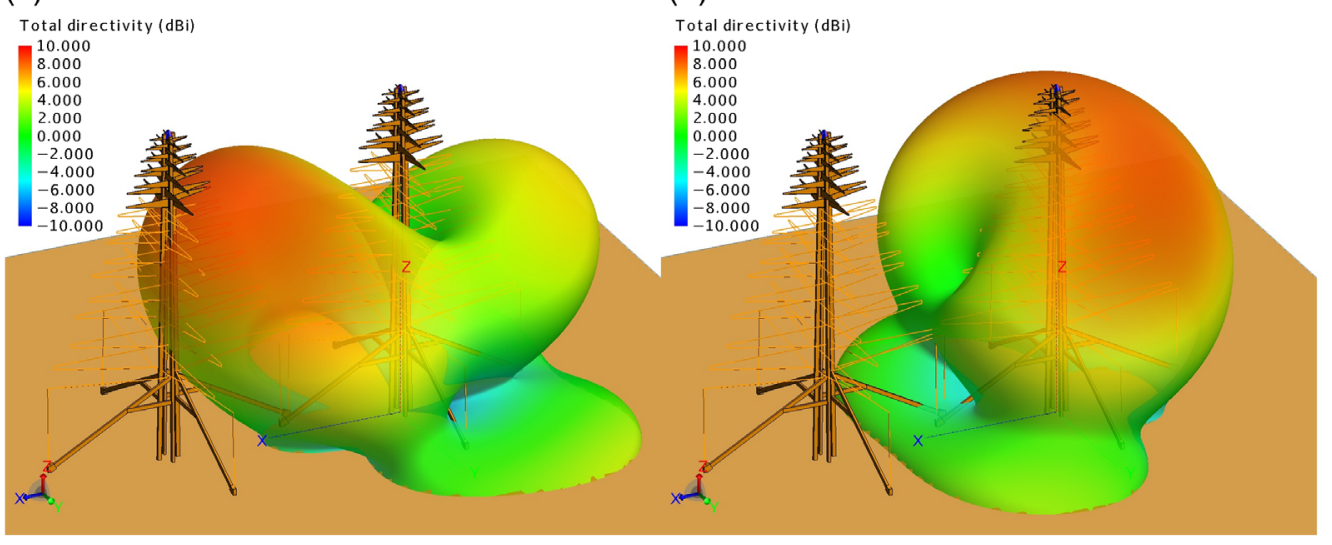

Fig. 5 3D antenna patterns for the $X$-polarized SKALA4.1 \#1 antenna (on the right side of each subplot) in the presence of the SKALA4.1 \#2 (on the left side). Four different frequencies were considered: (a) $54.5 \mathrm{MHz}$, (b) $57 \mathrm{MHz}$, (c) $77 \mathrm{MHz}$, and (d) $78 \mathrm{MHz}$.

The directivity at zenith versus frequency is shown in Fig. 8 (blue curves) for the $Y$ polarization of each antenna (with all other antenna ports closed on a 50-ohm load). As expected, the curves show quite different behaviors due to the different relative positions (and therefore different mutual coupling) with the other antennas. For instance, while the zenith directivity of antenna \#16 is almost unperturbed (quite flat between 50 and $100 \mathrm{MHz}$ ) because of the absence of other antennas in the $E$ plane, for antennas \#9 and \#10, the directivity is highly variable. Apart from the different responses of the antennas, sharp discontinuities around 55 and $77 \mathrm{MHz}$ appear for almost all antennas.

The next step is to compute the tile beam by summing up the individual electric fields with uniform phase and amplitude coefficients. For the tile, the effective area in the linear scale (equal to $G \lambda^{2} / 4 \pi$, with $G$ gain of the tile and $\lambda$ wavelength) is shown, in place of the directivity, to better emphasize the glitches in the response. Figure 9 shows the effective area at zenith for both polarizations of the tile (black and blue curves). This quantity is affected by the same structures at 55 and $77 \mathrm{MHz}$ already observed in the individual responses. This result confirms that the glitches are not cancelled by the randomized distribution of the tile.

An optimization of the antenna distribution can be defined exploiting the knowledge of the effects of mutual coupling as discussed in Sec. 2 for two antennas. In particular, exclusion zones have been created to avoid coupling of antennas aligned around the $E$ plane $( \pm 20 \mathrm{deg})$ and with an interdistance lower than $2.5 \mathrm{~m}$ (see the transparent blue areas of Fig. 7). Since the antennas are dual-polarized, this condition is applied for the $E$ plane of both polarizations. The layout in Fig. 7(a) clearly shows that almost all antennas do not satisfy this condition, with blue dots falling in the transparent blue areas. However, by applying shifts to nine antennas, it is possible to reconfigure the layout with all antennas outside the critical areas. The optimized layout is 
(a)

(b)

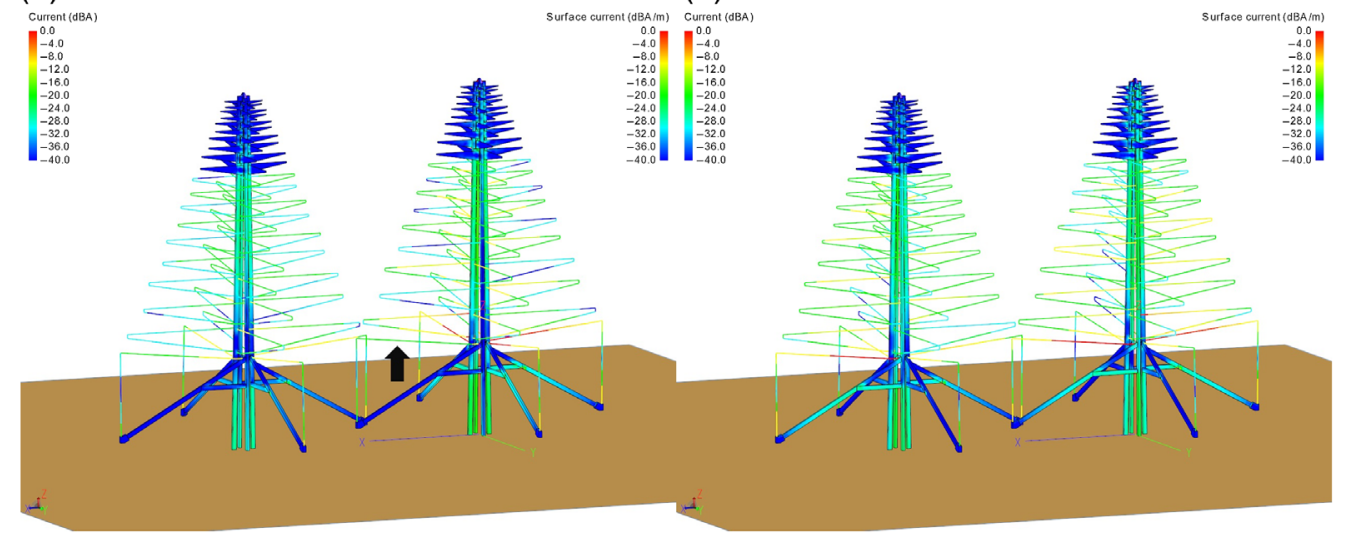

(c)

(d)

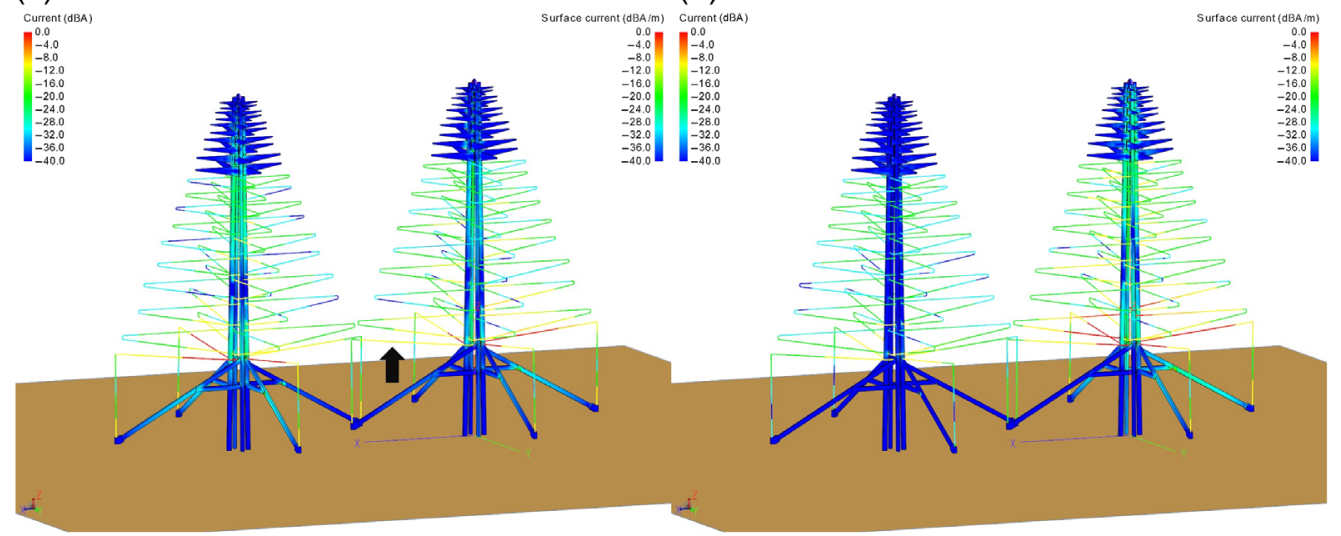

Fig. 6 Normalized linear and surface current distributions with the $X$-polarized SKALA4.1 $\# 1$ (right antenna) excited at four frequencies: (a) $54.5 \mathrm{MHz}$, (b) $57 \mathrm{MHz}$, (c) $77 \mathrm{MHz}$, and (d) $78 \mathrm{MHz}$. The black arrows in (a) and (c) identify, in the active antenna, the half of the bottom bow-tie dipole weakly excited at the resonant frequencies.
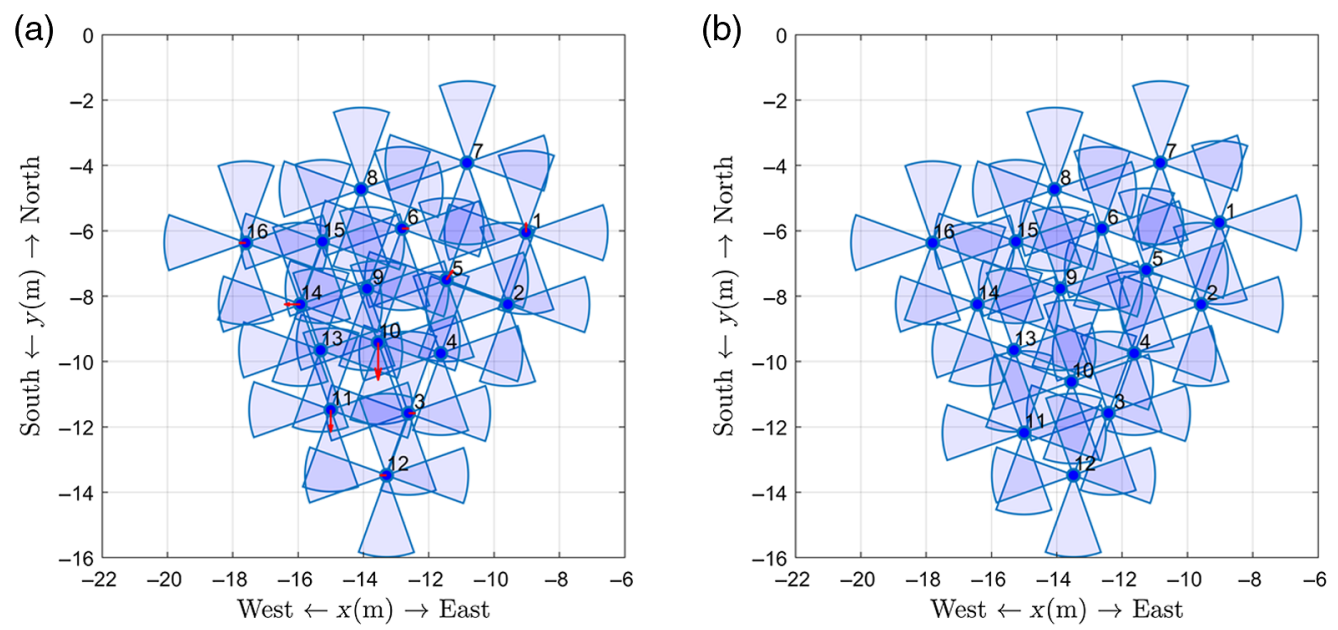

Fig. 7 Antenna distribution for the tile analyzed in this paper: (a) original layout as proposed in AAVS2 and (b) optimized layout considering exclusion zones. The exclusion zones (transparent blue areas) are given by the sector of circumferences of $2.5 \mathrm{~m}$ radius and \pm 20 deg with respect to the main axis. The arrows in (a) shows the translations applied to the antennas to obtain the layout of (b). 


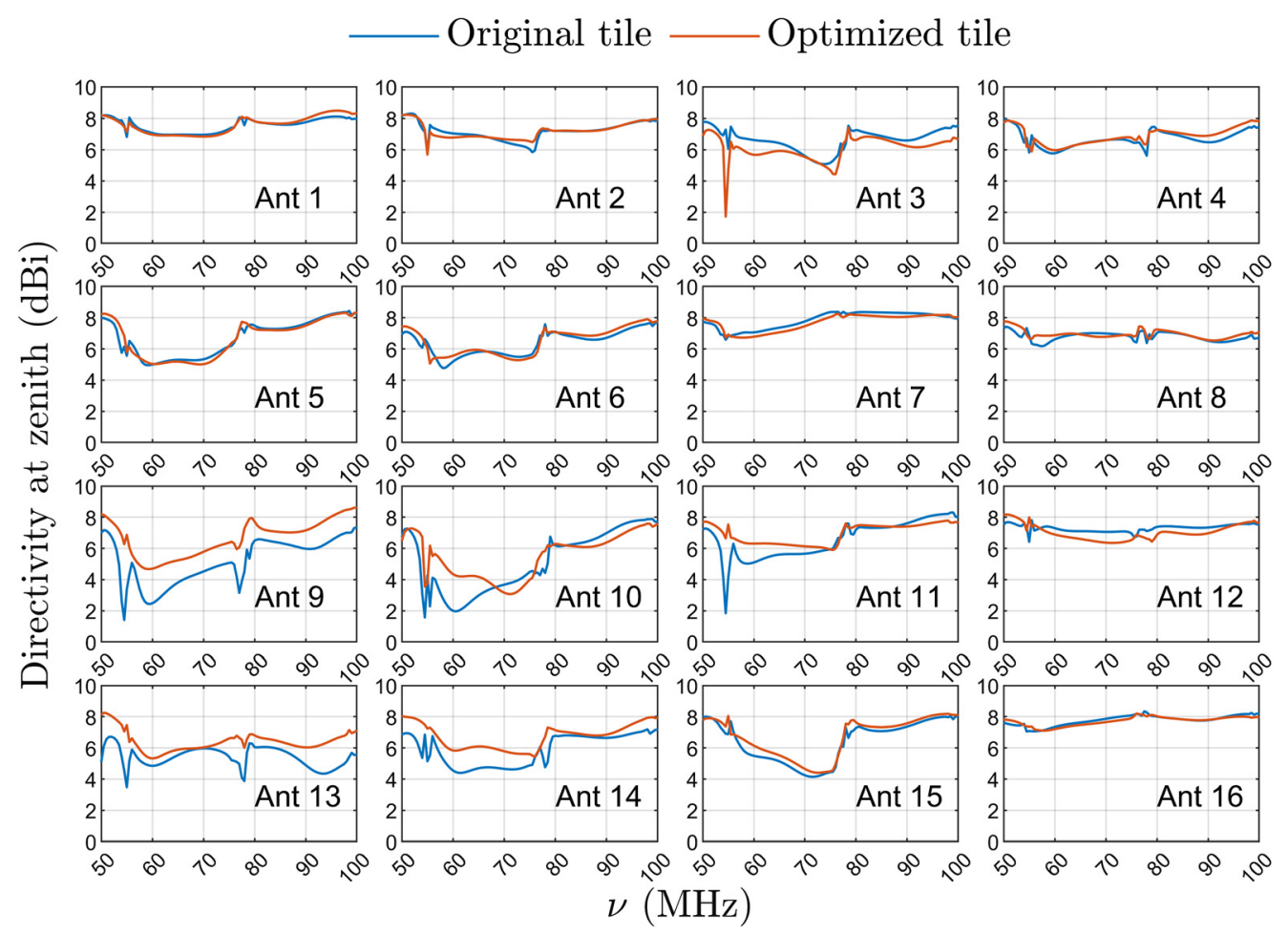

Fig. 8 Directivity at zenith in the frequency band 50 to $100 \mathrm{MHz}$ for each antenna composing one tile of AAVS2. The original tile layout is the blue curves; the optimized tile layout is the orange curves.

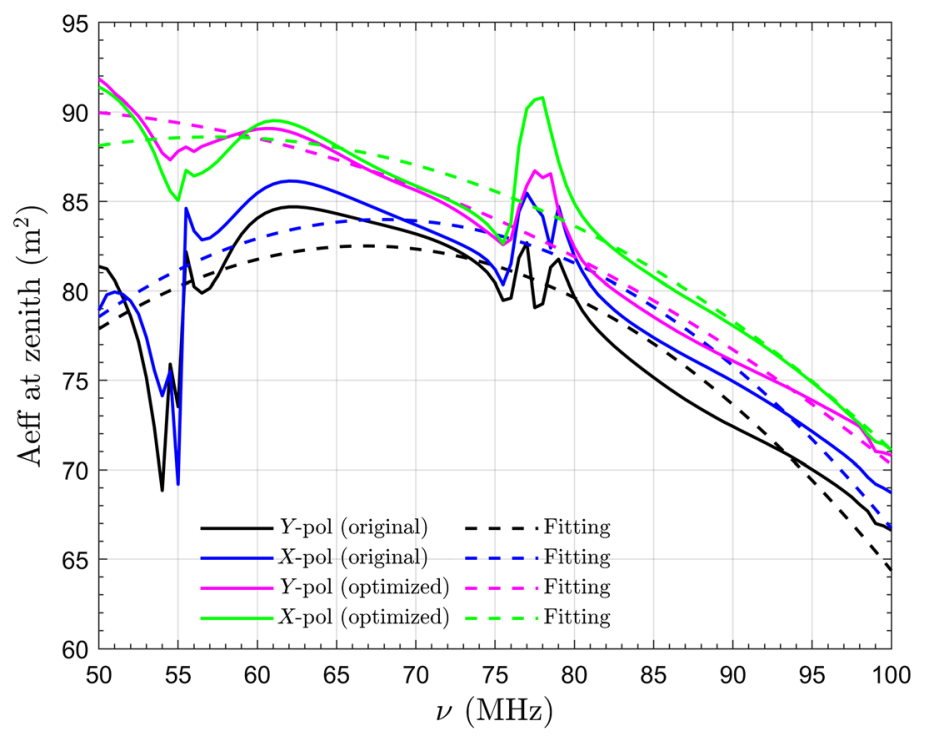

Fig. 9 Effective area at zenith (continuous curves) between 50 and $100 \mathrm{MHz}$ for both polarizations of a tile of 16 SKALA4.1 antennas. Blue and black curves refer to the nominal layout, whereas green and magenta curves are the optimized layout. Dashed curves represent the second-order polynomial fitting.

visible from Fig. 7(b). The amount of the shifts is kept as low as possible to strictly satisfy the condition given above: the translation of the nine antennas varies between $20 \mathrm{~cm}$ for antennas \#3, $\# 6$, \#12, and \#16 up to $1.2 \mathrm{~m}$ for antenna \#10. The tolerances in the antenna positioning on the field planned for SKA1-Low $( \pm 2.5 \mathrm{~cm})$ are given by the size of the pitch of the gridded mesh plate, and they are negligible as far as their impact on the mutual coupling among the antennas. 


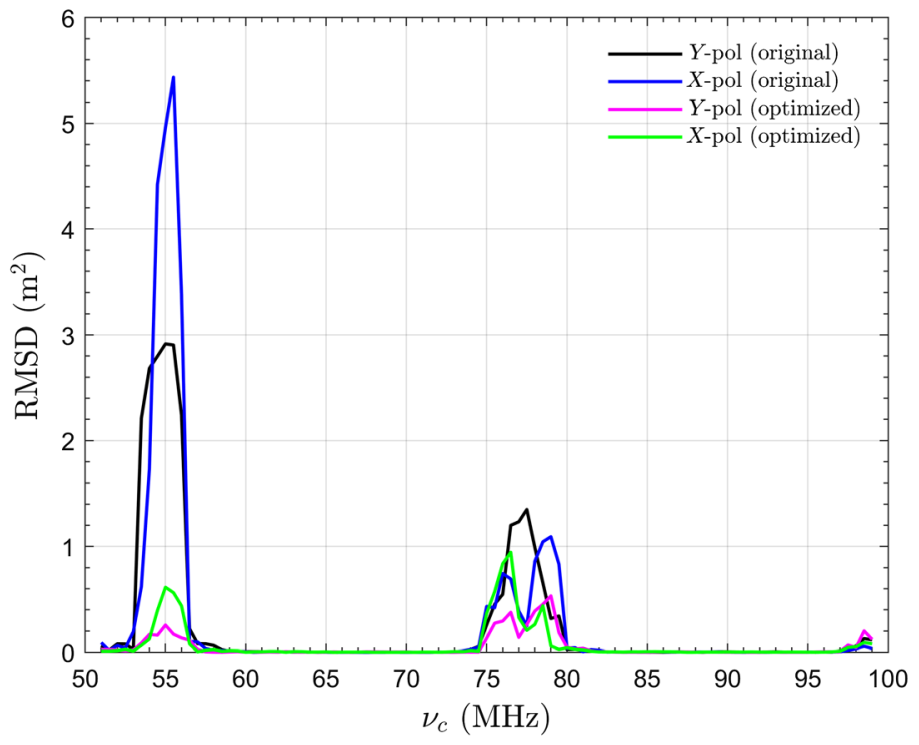

Fig. 10 Local residuals between second-order polynomial models and simulated effective areas shown in Fig. 9 considering narrow-band fittings $(2 \mathrm{MHz})$.

The optimized layout has been simulated, and overall, it shows less pronounced glitches in the zenith directivity of each element (see the orange curves of Fig. 8). This improvement is then confirmed by the effective area at zenith of the tile shown in Fig. 9 with magenta and green curves for the $Y$ and $X$ polarizations, respectively. In the 55-MHz region, the improvement in the smoothness behavior is remarkable with glitches almost completely removed. Despite the optimization being less effective at $77 \mathrm{MHz}$, the two-spike structure of the standard layout is replaced by a smoother single feature. The dashed curves of Fig. 9 show the second-order polynomial fitting of the four different effective areas computed in the whole frequency range of 50 to $100 \mathrm{MHz}$. The root-mean-square deviation (RMSD) of the difference between the polynomial fittings and the actual curves improves from 2.18 to $1.05 \mathrm{~m}^{2}$ for the $Y$ polarization and from 2.15 to $1.68 \mathrm{~m}^{2}$ for the $X$ polarization.

Finally, narrow-band second-order polynomial fittings are used to locally fit the simulated effective areas. The fittings are performed on adjacent partially overlapped spectral bands ( $2 \mathrm{MHz}$ wide each), and RMSDs are reported in Fig. 10 as a function of the central spectral channel of each subband. At $55 \mathrm{MHz}$, the RMSDs decrease considerably for both polarizations (by a factor 11 for the $Y$ polarization and 8 for the $X$ polarization), whereas, between 75 and $80 \mathrm{MHz}$, RMSDs decrease on average by a factor of 2 for both polarizations.

\section{Conclusions}

Although the isolated SKALA4.1 antenna does not feature glitches in the directivity response at low frequencies, the mutual coupling for a couple of antennas and for one tile of SKA1-Low with 16 antennas creates spectral regions where the current distribution on the SKALA4.1 antennas is strongly perturbed. Adopting exclusion zones in the antenna distribution, however, can improve the spectral smoothness below $100 \mathrm{MHz}$.

Based on the results discussed in this paper, the SKA Organization is considering an alternative layout to the currently pseudorandom distribution for the stations of SKA1-Low. ${ }^{16}$

The presence and frequency behavior of the presented resonances have been verified with two different full-wave simulation techniques, which can be considered to be sufficient validation for the proposed optimization strategy. Future works will include an experimental validation with both UAV-based antenna measurements already applied in the previous demonstrators of SKA1-Low ${ }^{17,18}$ and $S$-parameter measurements, the extension of the EM analysis to a full station of SKA1-Low, the assessment of the impact of these frequency structures on the 
science cases of SKA1-Low, and, if necessary, the study of calibration strategies to model the residual glitches.

\section{Acknowledgments}

The authors are grateful to Gianni Bernardi, Jader Monari, and Marco Schiaffino from INAFIRA and to Maria Grazia Labate from SKA Organization for their valuable contributions to this paper.

\section{References}

1. M. G. Labate et al., "The SKA low-frequency telescope: performance parameters and constraints on the array configuration," in 11th Eur. Conf. Antennas and Propag., Paris, France, pp. 2259-2263 (2017).

2. C. M. Trott and R. B. Wayth, "Spectral calibration requirements of radio interferometers for epoch of reionisation science with the SKA," Publ. Astron. Soc. Aust. 33, 1-13 (2016).

3. P. Bolli et al., "Test-driven design of an active dual-polarized log-periodic antenna for the square kilometre array," IEEE Open J. Antennas Propag. 1, 253-263 (2020).

4. E. de Lera Acedo, "SKALA: a log-periodic antenna for the SKA," in Proc. Int. Conf. Electromagn. Adv. Appl., Cape Town, South Africa, pp. 353-356 (2012).

5. E. de Lera Acedo et al., "Spectral performance of SKA Log-periodic Antennas I: mitigating spectral artefacts in SKA1-Low $21 \mathrm{~cm}$ cosmology experiments," Mon. Not. R. Astron. Soc. 469(3), 2662-2671 (2017).

6. G. Kyriakou et al., "Experimental verification of anomalous spectral features of SKALA4.1 antenna," in IEEE Int. Symp. Antennas and Propag. and USNC-URSI Radio Sci. Meeting, Marina Bay Sands, Singapore (2021).

7. C. Craeye and D. González-Ovejero, "A review on array mutual coupling analysis," Radio Sci. 46(2), 1-25 (2011).

8. C. M. Trott et al., "Spectral performance of square kilometre array antennas-II. Calibration performance," Mon. Not. R. Astron. Soc. 470(1), 455-465 (2017).

9. D. Davidson and D. Ung, "Spectral smoothness of embedded element patterns in the SKA-Low prototype station AAVS2: preliminary results," in IEEE Int. Symp. Antennas and Propag. and USNC-URSI Radio Sci. Meeting, Marina Bay Sands, Singapore (2021).

10. P. Di Ninni et al., "Mutual coupling analysis for a SKA1-Low station," in 13th Eur. Conf. Antennas and Propag., Krakow, Poland (2019).

11. J. Borg et al., "On-sky calibration of a SKA1-Low station in the presence of mutual coupling," Mon. Not. R. Astron. Soc. 496(1), 933-942 (2020).

12. D. González-Ovejero et al., "Non-periodic arrays for radio-astronomy applications," in IEEE Int. Symp. Antennas and Propag., Spokane, Washington, pp. 1762-1765 (2011).

13. P. Bolli et al., "Computational electromagnetics for the SKA-Low prototype station AAVS2," J. Astron. Telesc. Instrum. Syst. 8(1), 011017 (2022).

14. H. Bui-Van et al., "Fast and accurate simulation technique for large irregular arrays," IEEE Trans. Antennas Propag. 66, 1805-1817 (2018).

15. A. J. J. van Es et al., "A prototype model for evaluating SKA-Low station calibration," Proc. SPIE 11445, 1144589 (2020).

16. R. Braun, "SKA-Low station layouts revisted," SKA Technical Report n. SKAO-TEL0001829, rev. 01 (2021).

17. E. de Lera Acedo et al., "SKA aperture array verification system: electromagnetic modeling and beam pattern measurements using a micro UAV," Exp. Astron. 45(1), 1-20 (2018).

18. F. Paonessa et al., "SKA-Low prototypes deployed in Australia: synoptic of the UAV-based experimental results," Radio Sci. Lett. 2, 1-5 (2020).

Pietro Bolli received his laurea degree in electronic engineering and his $\mathrm{PhD}$ in computer science and telecommunications engineering from the University of Florence, Florence, Italy, in 1999 and 2003, respectively. In 2002, he started his professional career as a microwave engineer 
at the Italian National Institute for Astrophysics (INAF) conducting research in the field of technology applied to radio astronomy. He is currently a senior technologist at the INAF Arcetri Astrophysical Observatory. He is a co-author of about 140 scientific publications, which have appeared in international referred journals and conferences.

Mirko Bercigli received his laurea degree in electronic engineering from the University of Florence in 2000. Since September 2000, he has been employed in the Computational Electromagnetic Laboratory of IDS Ingegneria dei Sistemi S.p.A., as a system analyst in the field of electromagnetism. He is currently the head of the "Computational Electro Magnetic Engineering." He co-operates with several research centers such as the University of Florence, the Politecnico of Turin, the University of Pisa, the University of Siena, the University of l'Aquila, and the University of Rome in developing e.m. numerical methods. He also participated in several international programs supported by European Space Agency and European Community.

Paola Di Ninni received her physics degree in astrophysics and space physics from the University of Florence, Italy, in 2008, where she worked as a data analyst in the physics of matter from 2009 to 2013 and her PhD in experimental physics from the University of Siena, Italy, in 2017. She is currently working as a technologist in radio astronomy at the Arcetri Astrophysical Observatory of the National Institute of Astrophysics, Italy.

Lorenzo Mezzadrelli received his MSc degree in telecommunications engineering from the University of Bologna in 2004. Since 2004, he has been working in the R\&D Department of SIRIO Antenne as antenna designer and project manager. His activities are focused on design of base station and mobile antennas from $27 \mathrm{MHz}$ to $6 \mathrm{GHz}$. With Sirio Antenne, he is working on the Square Kilometre Array project (SKA), in particular on SKA-low antenna electrical and mechanical design.

Giuseppe Virone received his degree in electronic engineering (summa cum laude) and his $\mathrm{PhD}$ in electronics and communication engineering from the Politecnico di Torino, Turin, Italy, in November 2001 and 2006, respectively. He is currently a senior researcher at the Istituto di Elettronica e di Ingegneria Informatica e delle Telecomunicazioni (IEIIT) of the Italian National Research Council. He joined IEIIT as a research assistant in 2002. He coordinated more than 15 scientific projects funded by both the industry and other scientific research organizations and joined more than 30 research projects as a collaborator. He authored 43 journal papers, 134 conference papers, and 3 European patents. His activities concern the design, numerical analysis, and characterization of microwave and millimeter waveguide passive components for feed systems, antenna arrays, frequency selective surfaces, compensated dielectric radomes, and industrial sensing applications. 